\title{
Activity of Neurochemically Heterogeneous Dopaminergic Neurons in the Substantia Nigra during Spontaneous and Driven Changes in Brain State
}

\author{
Matthew T. C. Brown, Pablo Henny, J. Paul Bolam, and Peter J. Magill \\ Medical Research Council Anatomical Neuropharmacology Unit, University of Oxford, Oxford OX1 3TH, United Kingdom
}

\begin{abstract}
Dopaminergic neurons of the substantia nigra (SN) and ventral tegmental area (VTA) are collectively implicated in motor- and rewardrelated behaviors. However, dopaminergic SN and VTA neurons differ on several functional levels, and dopaminergic SN neurons themselves vary in their intrinsic electrical properties, neurochemical characteristics and connections. This heterogeneity is not only important for normal function; calbindin (CB) expression by some dopaminergic SN neurons has been linked with their increased survival in Parkinson's disease. To test whether the activity of CB-negative and CB-positive dopaminergic SN neurons differs during distinct spontaneous and driven brain states, we recorded single units in anesthetized rats before, during and after aversive somatosensory stimuli. Recorded neurons were juxtacellularly labeled, confirmed to be dopaminergic, and tested for CB immunoreactivity. During cortical slow-wave activity, the firing of most dopaminergic neurons was slow and regular/irregular and unrelated to cortical slow oscillations. During spontaneous cortical activation, dopaminergic SN neurons fired in a more regular manner, with fewer bursts, but did not change their firing rate. Regardless of brain state, CB-negative dopaminergic neurons fired significantly faster than CB-positive dopaminergic neurons. This difference in firing rate was not mirrored by different firing patterns. Most CB-negative and CB-positive dopaminergic neurons did not respond to the aversive stimuli; of those that did respond, most were inhibited. We conclude that CB-negative and CB-positive dopaminergic neurons exhibit different activities in vivo. Furthermore, the firing of dopaminergic SN neurons is brain state-dependent, and, unlike dopaminergic VTA neurons, they are not commonly recruited or inhibited by aversive stimuli.
\end{abstract}

\section{Introduction}

The midbrain dopaminergic system provides a modulatory input to diverse brain regions, including the cortex and basal ganglia, and plays a crucial role in the control of behavior. Midbrain dopaminergic neurons exhibit a continuum of patterned activity in vivo, which is often divided into two main modes; single-spike firing and burst firing (Grace and Bunney, 1984a,b). In response to rewarding stimuli that are greater than predicted, or to other salient stimuli, midbrain dopaminergic neurons in both the substantia nigra pars compacta $(\mathrm{SNc})$ and the ventral tegmental area (VTA) show transient increases in activity reminiscent of burst firing (Schultz and Romo, 1990; Redgrave et al., 1999; Hyland et al., 2002). Conversely, when an animal is presented with stimuli that are less rewarding than predicted, or even aversive, midbrain dopaminergic neurons are often inhibited (Mirenowicz and Schultz, 1996; Ungless et al., 2004).

\footnotetext{
Received Sept. 16, 2008; revised Dec. 10, 2008; accepted Jan. 8, 2009

This work was supported by the Medical Research Council (UK) and The Parkinson's Disease Society of the United Kingdom (Grant G-0601). M.T.C.B. was in receipt of a Medical Research Council studentship. We are grateful to M. Ungless, J. Mena-Segovia, J. Boyes, N. Mallet, and J. Moss for valuable scientific discussions. We also thank E. Norman, C. Francis, K. Whitworth, and B. Micklem ARPS for expert technical assistance.

Correspondence should be addressed to either Matthew T. C. Brown or Dr. Peter J. Magill, Medical Research Council Anatomical Neuropharmacology Unit, University of Oxford, Mansfield Road, Oxford OX1 3TH, UK, E-mail: mtc.brown@gmail.com or peter.magill@pharm.ox.ac.uk.

DOl:10.1523/JNEUROSCI.4423-08.2009

Copyright $\odot 2009$ Society for Neuroscience $\quad 0270-6474 / 09 / 292915-11 \$ 15.00 / 0$
}

There are several key differences between neurons of the VTA and $\mathrm{SNc}$ at the electrophysiological, neurochemical and anatomical levels. Dopaminergic VTA neurons show an increased tendency to fire in burst mode in vivo (Grenhoff et al., 1988; Zhang et al., 2008), and a higher proportion express the calcium-binding protein calbindin (CB) (Gerfen et al., 1985). Moreover, dopaminergic neurons of the VTA and SNc preferentially project to different regions of the forebrain (Fallon and Moore, 1978; Prensa and Parent, 2001; Björklund and Dunnett, 2007; Lammel et al., 2008), and afferents innervating the VTA and the SNc also differ (Smith et al., 1990; Korotkova et al., 2003; Balcita-Pedicino and Sesack, 2007; Mena-Segovia et al., 2008a). The neurons within the SNc itself are also heterogeneous. The CB-positive dopaminergic SNc neurons, which lie along the "dorsal tier" of the SNc (Gonzalez-Hernandez and Rodriguez, 2000), differ from CBnegative dopaminergic SNc neurons situated in the "ventral tier" in their intrinsic membrane properties (Neuhoff et al., 2002), dendritic organization and axonal targets (Fallon et al., 1978; Gerfen et al., 1987). Moreover, CB-positive dopaminergic neurons are less susceptible to degeneration in Parkinson's disease (PD) and its animal models (Yamada et al., 1990).

The differences between dopaminergic neurons of the VTA and $\mathrm{SNc}$, and the heterogeneity within the $\mathrm{SNc}$, imply functional divisions of the midbrain dopaminergic system, and therefore different roles in the modulation of behavior. For example, these differences may be reflected in distinct patterns of activity 
during different brain states or their responsiveness to aversive stimuli. In addition, the possibility that SNc contains a population of nondopaminergic neurons that are electrophysiologically similar to dopaminergic neurons, as described in the VTA (Cameron et al., 1997; Ungless et al., 2004), adds another level of complexity and serves to highlight the need for unequivocal determination of the neurochemical phenotype of recorded neurons.

To define the activity of identified substantia nigra (SN) dopaminergic neurons in relation to both spontaneous and "driven" brain states, we combined extracellular unit and electrocorticogram recordings in anesthetized rats, and tested neuronal responses to aversive somatosensory stimuli. After physiological characterization, we labeled the recorded neurons using the juxtacellular method and identified their neurochemical properties using immunohistochemistry.

\section{Materials and Methods}

Electrophysiological recordings. Experimental procedures were performed on adult male Sprague Dawley rats (Charles River) and were conducted in accordance with the Animals (Scientific Procedures) Act, 1986 (United Kingdom), and the European Communities Council Directive (86/609/EEC).

Electrophysiological recordings were made in 42 rats (240-370 g). Anesthesia was induced with isoflurane (Isoflo, Schering-Plough) and maintained with urethane (1.3 g/kg, i.p.; ethyl carbamate, Sigma), and supplemental doses of ketamine (30 mg/kg, i.p.; Ketaset, Willows Francis) and xylazine (3 mg/kg, i.p.; Rompun, Bayer), as described previously (Magill et al., 2001). All wound margins were infiltrated with the local anesthetic bupivacaine $(0.75 \% \mathrm{w} / \mathrm{v}$; Astra) and corneal dehydration was prevented with application of Hypromellose eye drops (Norton Pharmaceuticals). Animals were then placed in a stereotaxic frame. Body temperature was maintained at $37 \pm 0.5^{\circ} \mathrm{C}$ using a homeothermic heating device (Harvard Apparatus).

Anesthesia levels were assessed by examination of the electrocorticogram (ECoG, see below), and by testing reflexes to a cutaneous pinch or gentle corneal stimulation. The electrocardiogram (ECG) and respiration rate were also monitored constantly to ensure appropriate anesthetic levels (see below). Saline solution $(0.9 \% \mathrm{w} / \mathrm{v} \mathrm{NaCl})$ was applied to all areas of exposed cortex to prevent dehydration.

The ECoG was recorded via a $1 \mathrm{~mm}$ diameter steel screw juxtaposed to the dura mater above the right frontal cortex (AP: $+2.7 \mathrm{~mm}$, ML: $2.0 \mathrm{~mm}$ in relation to bregma (Paxinos and Watson, 1986)), and was referenced against another screw implanted in the skull above the cerebellum. Raw ECoG was bandpass filtered $(0.3-1500 \mathrm{~Hz},-3 \mathrm{~dB}$ limits $)$ and amplified (2000×, DPA-2FS filter/amplifier; Scientifica) before acquisition. The ECG was differentially recorded via two silver wires inserted into the skin of the left forelimb and hindlimb. Raw ECG was bandpass filtered (10$100 \mathrm{~Hz}$ ) and amplified (5000×, DPA-2FS; Scientifica) before acquisition. A discrete craniotomy was performed above the right and/or left $\mathrm{SN}$, and the dura mater removed for insertion of recording electrodes. Extracellular recordings of action potentials of $\mathrm{SN}$ neurons were made using $10-25 \mathrm{M} \Omega$ glass electrodes (tip diameter $\sim 1.5 \mu \mathrm{m}$ ), which contained saline solution $(0.5 \mathrm{M} \mathrm{NaCl})$ and Neurobiotin $(1.5 \% \mathrm{w} / \mathrm{v}$, Vector Laboratories). Electrode signals were amplified $(10 \times)$ through the active bridge circuitry of an Axoprobe-1A amplifier (Molecular Devices Corp.), bifurcated, then differentially filtered to extract local field potentials (LFPs) and unit activity. The LFPs were recorded after further amplification (100×; DPA-2FS; Scientifica) and "wideband" filtering (between 0.3 and $5000 \mathrm{~Hz}$; DPA-2FS; Scientifica). Single units were recorded after alternating current coupling, further amplification (100×; DPA-2FS; Scientifica), and standard bandpass filtering (between 300 and $5000 \mathrm{~Hz}$; DPA-2FS; Scientifica). A Humbug (Quest Scientific) was used in place of a traditional "notch" filter to eliminate mains noise at $50 \mathrm{~Hz}$ (Brown et al., 2002). Spikes were often several millivolts in amplitude and always exhibited an initial positive deflection. Neuronal firing was recorded during slow-wave activity (SWA), which accompanies deep anesthesia and is similar to activity observed during natural sleep, and during epi- sodes of cortical activation, which contain patterns of activity that are more analogous to those observed during the awake, behaving state (Steriade, 2000). Transition from SWA to the activated brain state is exemplified by obliteration of the cortical slow oscillation $(\sim 1 \mathrm{~Hz})$, as well as $\delta(1-4 \mathrm{~Hz})$ and spindle $(7-14 \mathrm{~Hz})$ oscillations (Steriade, 2000).

After recordings of spontaneous activity, the responses of individual neurons and the ECoG to two standard types of aversive somatosensory stimuli (a pinch or an electrical stimulus applied to the hindpaw contralateral to the recording site) were characterized. Pinches of $15 \mathrm{~s}$ duration were delivered using pneumatically driven forceps that consistently delivered a standard pressure of $183 \mathrm{~g} / \mathrm{mm}^{2}$ (Pollard, 2000; Ungless et al., 2004), which has previously been shown to exceed the threshold of pain in behaving rats (Cahusac et al., 1990). Electrical stimuli (single current pulses of $5 \mathrm{~mA}$ intensity and $2 \mathrm{~ms}$ duration) were delivered at $0.5 \mathrm{~Hz}$ (Coizet et al., 2006) for 100 trials. The timings of stimuli delivery were precisely controlled by an external pulse generator (Master-8; A.M.P.I.). The animals did not exhibit a marked change in ECG or respiration rate, nor a hindpaw withdrawal reflex, in response to the pinch or the electrical stimuli, thus indicating anesthesia was adequate throughout recordings.

Juxtacellular labeling of single neurons. To locate the recorded neurons and enable an analysis of their morphological and neurochemical properties, they were then labeled with Neurobiotin (Vector Laboratories) by the juxtacellular method (Pinault, 1996; Magill et al., 2000). Briefly, the electrode was advanced slowly toward the neuron while a microiontophoretic current was applied (1-10 nA positive current, $200 \mathrm{~ms}$ duration, $50 \%$ duty cycle). The optimal position of the electrode was identified when the firing pattern of the neuron was robustly modulated by the current injection. It was necessary to modulate the neuronal firing by the microiontophoretic current for at least $2 \mathrm{~min}$, but preferably longer, to obtain reliable labeling. The Neurobiotin was then left to transport along neuronal processes for up to $12 \mathrm{~h}$. After the recording and labeling sessions, the animals were given a lethal dose of ketamine $(150 \mathrm{mg} / \mathrm{kg})$ and perfused via the ascending aorta with $100 \mathrm{ml}$ of $0.01 \mathrm{M} \mathrm{PBS}$ at $\mathrm{pH} 7.4$, followed by $300 \mathrm{ml}$ of $0.1 \% \mathrm{w} / \mathrm{v}$ glutaraldehyde and $4 \% \mathrm{w} / \mathrm{v}$ paraformaldehyde in $0.1 \mathrm{~m}$ phosphate buffer, $\mathrm{pH} 7.4$, and then by $100 \mathrm{ml}$ of PBS. Brains were then left in PBS or fixative solution at $4^{\circ} \mathrm{C}$ until they were sectioned $24-72 \mathrm{~h}$ later.

Histochemistry. The fixed brains were sectioned at $60 \mu \mathrm{m}$ in the parasagittal plane on a vibrating blade microtome (VT1000S; Leica Microsystems,). Selected tissue sections were then incubated $\left(4^{\circ} \mathrm{C}, 2\right.$ nights) in PBS containing primary antibodies raised against tyrosine hydroxylase (TH, mouse anti-TH; 1:1000, Sigma) and calbindin (rabbit anti-CB; 1:5000, Swant, or goat anti-CB; 1:500, Santa Cruz Biotechnology). Triton $\mathrm{X}-100(0.3 \% \mathrm{v} / \mathrm{v})$ and $1 \% \mathrm{w} / \mathrm{v}$ bovine serum albumin (Sigma) were included in the incubation mixture. Fluorescent conjugates were used to visualize Neurobiotin (streptavidin-CY3; 1:1000, $4^{\circ} \mathrm{C}$, overnight; Zymed), TH immunoreactivity (AlexaFluor 488-conjugated donkey anti-mouse; $1: 1000,4^{\circ} \mathrm{C}$, overnight; Invitrogen) and $\mathrm{CB}$ immunoreactivity (CY5-conjugated donkey anti-rabbit or CY5-conjugated donkey antigoat; $1: 500,4^{\circ} \mathrm{C}$, overnight; Jackson Immunoresearch Laboratories). Sections were then mounted on slides for viewing with a conventional epifluorescence microscope (Leica Microsystems), or a laser-scanning confocal microscope (Zeiss).

After analysis in the fluorescence microscope, standard histochemical techniques were used to visualize the Neurobiotin-filled neurons with a permanent peroxidase reaction product for light microscopy. Unless otherwise stated, histochemical incubations were performed at room temperature. Sections were washed in PBS and incubated overnight $\left(4^{\circ} \mathrm{C}\right)$ in avidin-biotin peroxidase complex (ABC Elite; 1:100; Vector) in PBS (with or without $0.3 \% \mathrm{v} / \mathrm{v}$ Triton X-100) and $1 \% \mathrm{w} / \mathrm{v}$ bovine serum albumin. After washing, the sections were incubated in hydrogen peroxide $(0.002 \% \mathrm{w} / \mathrm{v} ;$ Sigma $)$ and diaminobenzidine tetrahydrochloride (DAB; $0.025 \% \mathrm{w} / \mathrm{v}$; Sigma) in the presence of nickel ammonium sulfate $(0.5 \% \mathrm{w} / \mathrm{v}$; Sigma) dissolved in Tris buffer $(0.05 \mathrm{M}, \mathrm{pH} 8.0)$ for $5-30 \mathrm{~min}$. Neurobiotin-filled neurons were labeled intensely with an insoluble, black/blue precipitate. Sections containing the cell body were then incubated in rabbit anti-mouse IgG (1:200, 1 h; Dako), washed with PBS, and incubated in mouse peroxidase anti-peroxidase (PAP; 1:100, $2 \mathrm{~h}$; Dako). After this, the DAB procedure was repeated (omitting the nickel ammo- 
nium sulfate, and DAB prepared in Tris buffer $\mathrm{pH}$ 7.6), so that the THimmunoreactive (dopaminergic) neurons of the substantia nigra were labeled with a brown precipitate.

Electrophysiological data analysis. All biopotentials were digitized online using a Power 1401 Analog-Digital converter (Cambridge Electronic Design) and a PC running Spike2 acquisition and analysis software (version 5.15; Cambridge Electronic Design). Unit activity, LFPs and the ECoG were sampled at $16.7 \mathrm{kHz}, 16.7 \mathrm{kHz}$ and $5.6 \mathrm{kHz}$, respectively. Data from the recording sessions were inspected visually and epochs of stationary cortical activity were identified. A portion of the coincident spike train composed of 150 spikes was then isolated and used for our standard statistical analyses. Measurements of the extracellular action potential waveform were taken from these data epochs, as were spontaneous firing rate $(\mathrm{Hz})$ and the coefficient of variation of spiking $(\mathrm{CV}$, a standard measure of regularity). Autocorrelograms of unit activity (50 ms bins) and ECoG power spectra (frequency resolution of $0.17 \mathrm{~Hz}$ ) were constructed using standard procedures in Spike2. To test whether dopaminergic neurons engaged in bursting activity, the classical criteria outlined by Grace and Bunney were used (Grace and Bunney, 1984a). Briefly, bursts were defined as beginning when 2 action potentials occurred within $80 \mathrm{~ms}$ of each other, and ending when an action potential did not occur for $160 \mathrm{~ms}$. Previous studies have additionally categorized the spontaneous firing patterns of dopaminergic neurons into 3 groups: "pacemaker", "random" or "bursty" (Tepper et al., 1995; Paladini and Tepper, 1999). When possible, we tested our neurons according to this additional classification scheme. To achieve this, autocorrelograms (5 ms bins, plotted over $2 \mathrm{~s}$ ) were constructed from data epochs containing $\geq 1000$ spikes. Cells that exhibited 3 or more equally spaced peaks in the autocorrelogram were classified as pacemakers, cells that exhibited a distinct initial peak followed by a return to steady state were classified as bursty, and all others were classified as random (Tepper et al., 1995; Paladini and Tepper, 1999).

The slow $(\sim 1 \mathrm{~Hz})$ oscillation that dominates SWA, as recorded in ECoGs or basal ganglia LFPs, does not closely approximate a sinusoidal waveform, and contains active and inactive components of varied shape and duration. This makes detecting the peaks (or troughs) of this oscillation difficult, and also limits the utility of several analyses that are commonly used for defining the relationships between unit activity and ECoG/LFP oscillations (e.g., spike-triggered waveforms or circular statistics). Thus, we used an alternative method of quantifying the temporal relationship between the cortical slow oscillation and unit activity (Mallet et al., 2008). Single-unit activity was converted so that the "time stamp" of each spike was represented by a single digital event (Spike2). Raw ECoGs were initially down-sampled to $1.79 \mathrm{kHz}$ off-line, high-pass filtered at $0.3 \mathrm{~Hz}$ to remove any slow drift in DC potential, and then bandpass filtered at $0.4-1.6 \mathrm{~Hz}$ to isolate the cortical slow oscillation (MATLAB). The zero-voltage crossings of this filtered ECoG signal were then used to define the start and end points of active (and inactive) components. However, active and inactive components were only considered as such after thresholding for voltage (amplitude difference between components of $\geq 0.3 \mathrm{mV}$ ) and power (ratio of component power, which takes into account the signal energy over time, was $>0.15$ ). Thus, only robust slow oscillations were analyzed. Moreover, active components were only accepted if they were preceded or followed by an inactive component and vice versa. After defining active and inactive components of ECoGs, coincident spikes were automatically assigned to one of 14 bins (7 bins each for active and inactive components: MATLAB). Spike counts per bin across all accepted oscillation components were then normalized (by converting to firing rate) to take into account the variable durations of active and inactive components, and then displayed in an "activity histogram" (Fig. 1). For statistical definition of relationships between unit activity and the cortical slow oscillation, we used Pearson's $\chi^{2}$ test (Excel, Microsoft) to assess the goodness of fit of the observed firing, as indicated in the activity histogram, to the expected firing. The null hypothesis, which dictated the expected firing, was that firing during the active component was the same as that during the inactive component, i.e., that unit activity was not modulated in time with the slow oscillation. Significance for the $\chi^{2}$ test was set at $p<0.05$.

To quantify the responses of dopaminergic neurons to the pinch stim- ulus, a baseline of spontaneous unit activity recorded for $30 \mathrm{~s}$ immediately preceding pinch onset was first established. This baseline was compared with the activity both during, and $15 \mathrm{~s}$ immediately after, the pinch. The mean firing rate and SD of activity during the baseline period were calculated. Firing rate was plotted against time ( $1 \mathrm{~s}$ bins), and the number of bins above and below 2 SDs from the baseline mean rate were calculated. A neuron was defined as significantly inhibited or excited by a pinch stimulus if 2 consecutive histogram bins within the $15 \mathrm{~s}$ stimulus period lay outside 2 SDs from the baseline mean rate. A neuron was defined as exhibiting a rebound excitation if 2 consecutive histogram bins lay above 2 SDs from the mean up to $5 \mathrm{~s}$ after stimulus offset. To quantify the responses of dopaminergic neurons to the electrical stimulus, artifacts were first removed from the spike train (Spike2, custom script), and then peri-stimulus time interval histograms (PSTHs) of unit activity were constructed from $0.5 \mathrm{~s}$ before to $1 \mathrm{~s}$ after the stimulus delivery (20 ms bins) (Coizet et al., 2006). A neuron was defined as exhibiting a significant response to the electrical stimulus if 2 consecutive PSTH bins lay ouside 2 SDs from the baseline mean rate (measured as the $500 \mathrm{~ms}$ of activity before the stimulus). To assess the influence of the electrical stimulus on cortical activity, peristimulus evoked potentials (as recorded in the ECoG) were calculated.

Statistical analysis. The single-sample Kolmogorov-Smirnov test was used to judge whether data sets were normally distributed ( $p \leq 0.05$ to reject). Because some data sets were not normally distributed, we used nonparametric statistical testing throughout (SigmaStat, Systat Software Inc.). The Mann-Whitney $U$ test was used for comparisons of unpaired data, whereas the Wilcoxon signed rank test was used to compare paired data sets. Significance for all statistical tests was set at $p<0.05$ unless noted otherwise.

\section{Results}

\section{Cortical slow-wave activity}

Prevailing activity in frontal cortex was assessed from ECoG recordings. As described previously (Steriade et al., 1993; Magill et al., 2001), urethane anesthesia was typically accompanied by a large amplitude $(>400 \mu \mathrm{V})$, slow $(\sim 1 \mathrm{~Hz})$ oscillation in the frontal ECoG. Higher-frequency activity of smaller amplitude $(<200$ $\mu \mathrm{V}$ ), including spindle activity, was often superimposed on specific portions (the "peaks") of the cortical slow oscillation. These parts of the slow oscillation are associated with synchronous spike discharges in cortical projection neurons, and will be referred to as the "active component." Portions of the slow oscillation that do not contain this smaller amplitude, higher frequency activity (that is, the "troughs"), will be referred to as the "inactive component."

\section{Activity of identified dopaminergic neurons in the substantia nigra during cortical SWA}

We recorded the spontaneous action potential discharges of putative dopaminergic neurons (unit activity) in the $\mathrm{SN}$, in conjunction with cortical activity, in anesthetized rats. Only those neurons later confirmed to be dopaminergic by their expression of TH ( $n=56$, Fig. $1 A, B, \mathrm{D})$ were analyzed further and included here. Of the 56 neurons identified as dopaminergic, 44 were recorded during the presence of a robust cortical slow oscillation. Typical electrophysiological properties of dopaminergic neurons during periods of cortical SWA included a slow firing rate of $\sim 2-5 \mathrm{~Hz}$ (Fig. $1 A, B$ ), and a firing pattern that fluctuated between regular and irregular activity (Fig. $1 A, B$ ). Bursting activity, as defined by widely used criteria (Grace and Bunney, 1984a), occurred in a minority of recorded neurons ( 11 of 44 ); these bursts were exemplified by spike height accommodation and increasing interspike interval. In cases when the cortical slow oscillation remained sufficiently stationary, and $\geq 1000$ coincident spikes were recorded ( $n=27$ neurons), we performed the additional 

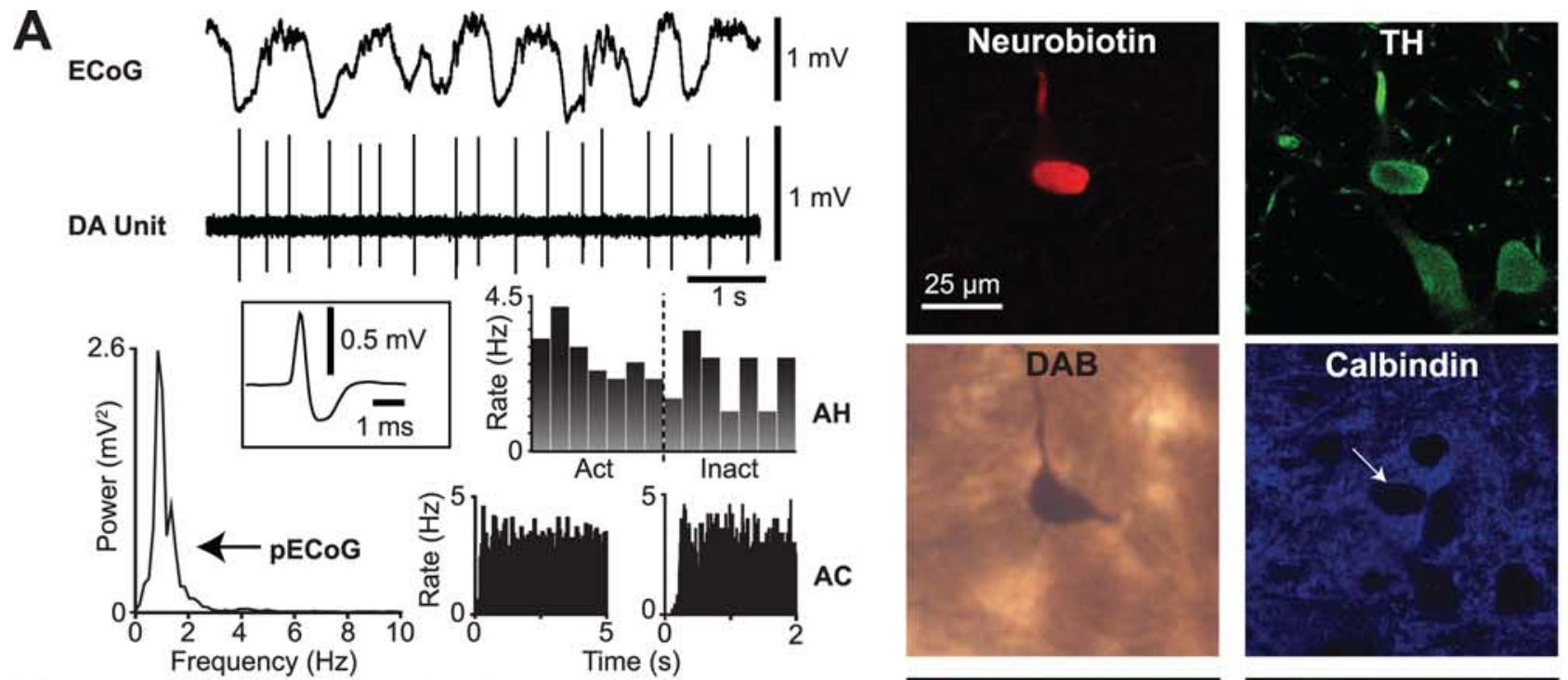

B
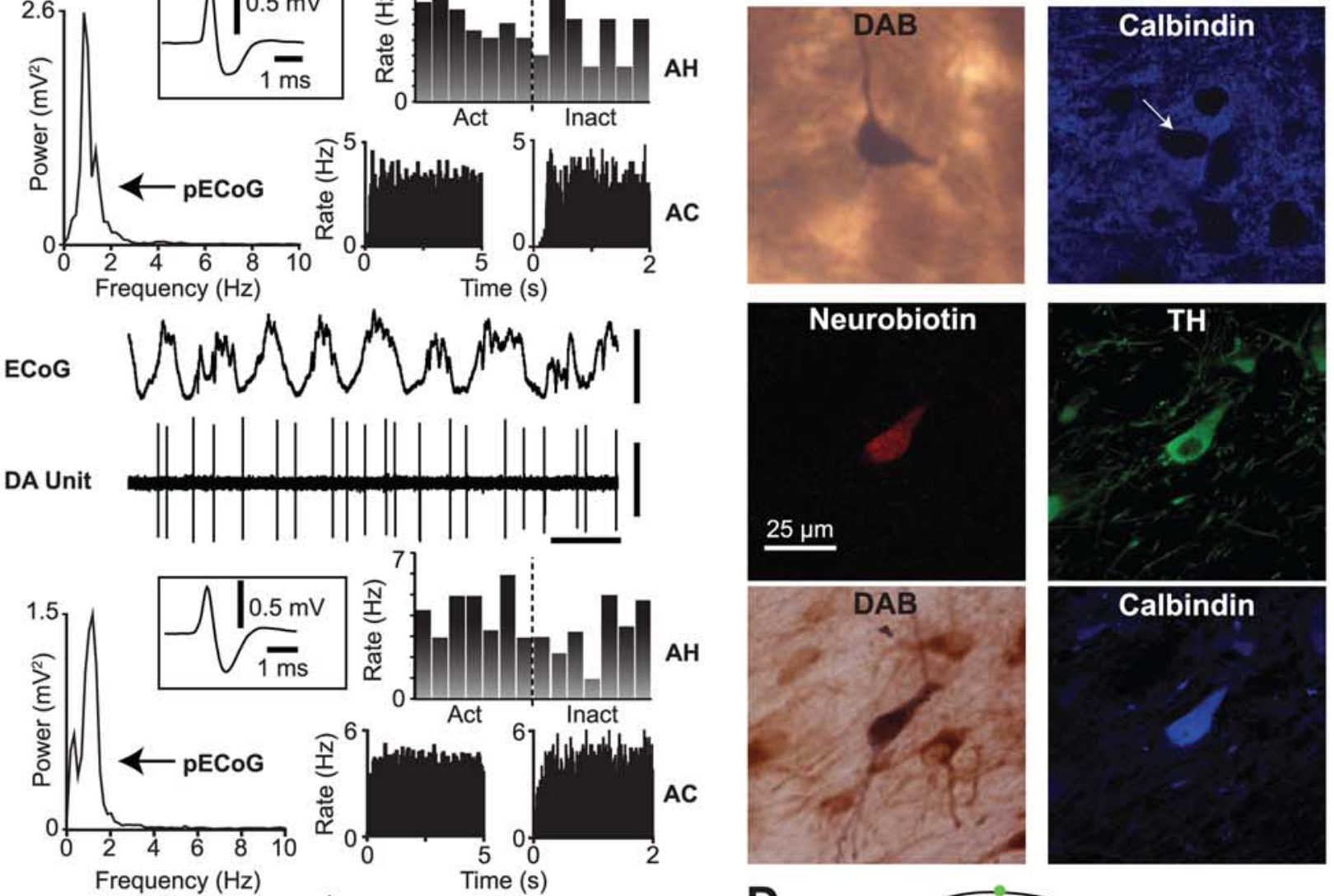

C
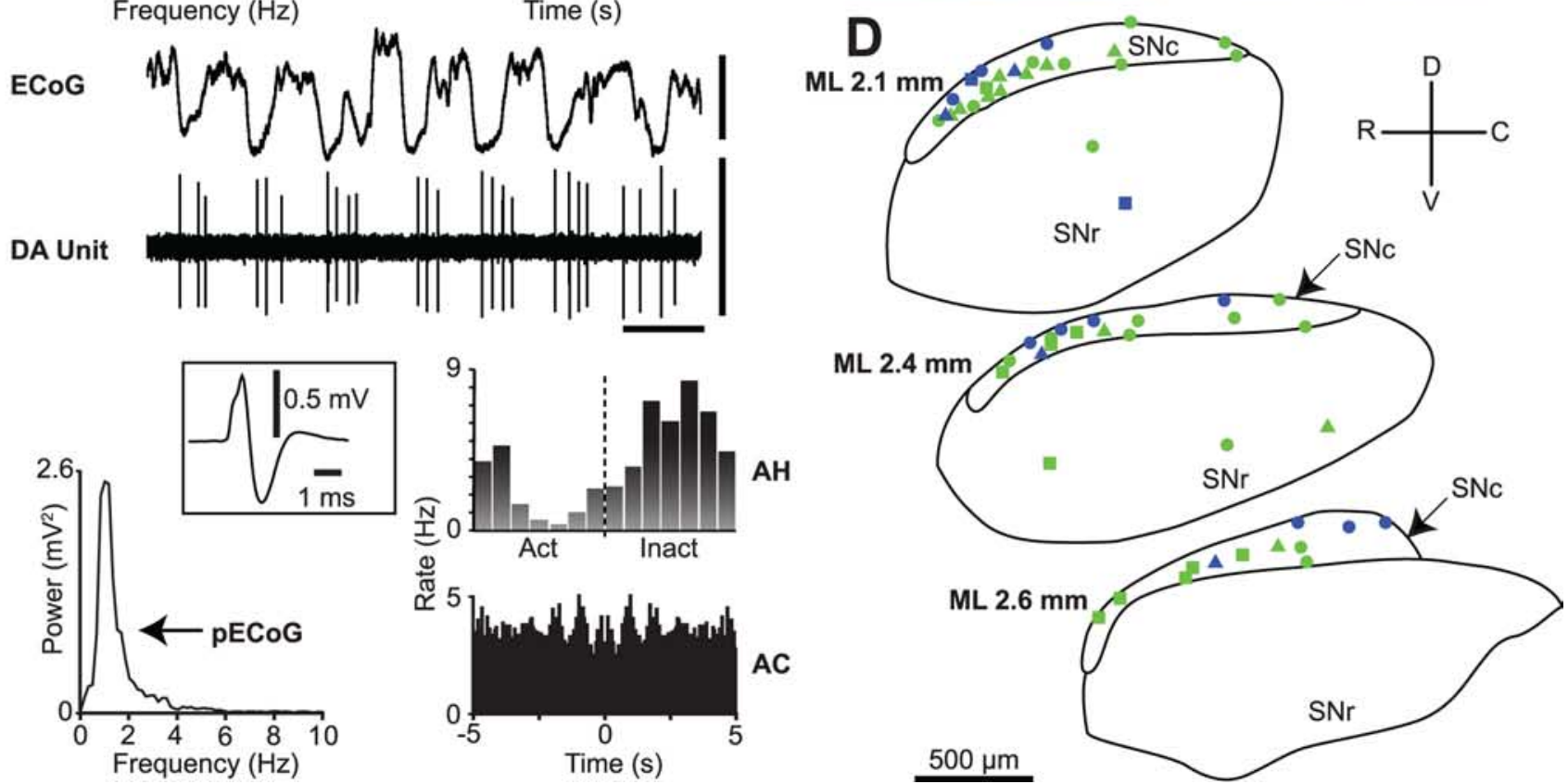

Figure 1. Spontaneous activity of identified dopaminergic neurons in the substantia nigra. $\boldsymbol{A}$, Recording of spontaneous unit activity (DA Unit) of a typical calbindin-negative dopaminergic neuron during robust slow-wave activity recorded in the ECOG. Cortical activity is dominated by a $\sim 1 \mathrm{~Hz}$ oscillation, as shown in the ECoG power spectrum (pECOG). Unit activity is irregular, as shown by a relatively flat autocorrelogram (AC; left in 50 ms bins, right in 5 ms bins), and the neuron did not fire preferentially during either the active (Act) or inactive (Inact) component of the (Figure legend continues.) 
A

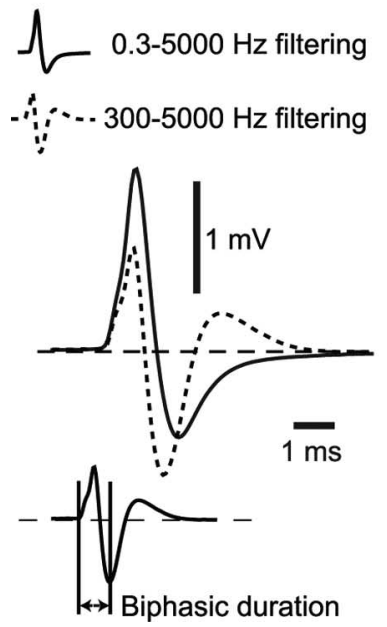

B

$300-5000 \mathrm{~Hz}$ filtering

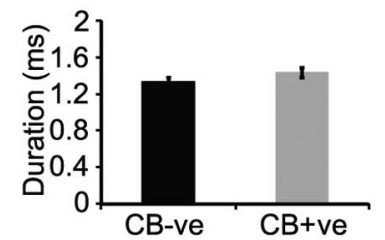

\section{C}

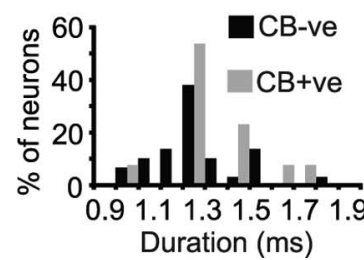

$0.3-5000 \mathrm{~Hz}$ filtering
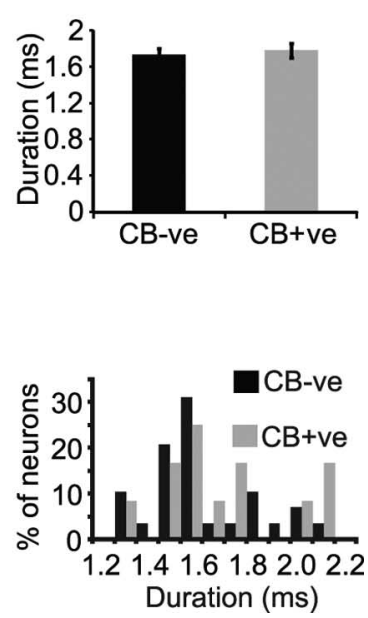

Figure 2. Effect of analog filtering on the action potential waveforms of identified dopaminergic substantia nigra neurons. $A$, Waveform averages of the same 150 action potentials of a single neuron filtered with either standard bandpass settings ( 300 $5000 \mathrm{~Hz}$, dashed line) or wideband settings $(0.3-5000 \mathrm{~Hz}$, solid line). Measurement used for biphasic action potential duration shown in bottom right. $\boldsymbol{B}$, Mean biphasic action potential durations of calbindin-negative (CB-ve) and calbindin-positive (CB +ve) dopaminergic neurons according to standard bandpass filtering (left) and wideband filtering (right). C, Population histograms for biphasic action potential durations filtered with either standard settings (left) or wideband settings (right).

firing pattern analysis and found that 8 identified dopaminergic neurons were classified as pacemaker, 16 as random and 3 as bursty (supplemental Fig. 1, available at www.jneurosci.org as supplemental material). Most dopaminergic neurons did not preferentially fire during either component of the ongoing cortical slow oscillations (Fig. 1A,B). A small subset of neurons ( 3 of 44) fired in an oscillatory pattern at $\sim 1 \mathrm{~Hz}$, and their firing was significantly modulated in time with the cortical slow oscillation (Fig. 1C). These 3 neurons preferentially fired during the inactive component of the cortical oscillation (Fig. 1C). Identified dopaminergic neurons in the SNc $(n=51)$ were located throughout the nucleus, as visualized by permanent staining of Neurobiotin and TH for light microscopic evaluation (Fig. 1). However, 5 dopaminergic neurons were located within the substantia nigra pars reticulata $(\mathrm{SNr})$ (Fig. 1D), into which columns of dopaminergic neurons extend from the SNc (Gonzalez-Hernandez and Rodriguez, 2000). These dopaminergic neurons displayed firing rates and patterns similar to dopaminergic neurons located in the

(Figure legend continued.) cortical slow oscillation (activity histogram, AH). Average action potential waveform (filter settings $300-5000 \mathrm{~Hz}$ ) of the recorded neuron is shown in the inset. After recording, this neuron was revealed to be a calbindin-negative dopaminergic neuron, as shown in the digital micrographs of Neurobiotin, tyrosine hydroxylase (TH), and calbindin reactivity (arrow points to neuron). Neurobiotin was later visualized with diaminobenzidine (DAB) to form a permanent reaction product for light microscopy. $\boldsymbol{B}$, A typical calbindin-positive dopaminergic neuron. Note the similarity in electrophysiological characteristics between units in $\boldsymbol{A}$ and $\boldsymbol{B}$. C, An identified calbindin-negative dopaminergic neuron that showed oscillatory activity at $\sim 1 \mathrm{~Hz}$, as shown by the peaks in the ACs. This neuron preferentially fired in time with the inactive component of the cortical slow oscillation, as shown in the AH. D, Schematic of sagittal sections of the substantia nigra pars compacta (SNc) and substantia nigra pars reticulata ( $\mathrm{SNr}$ ) showing approximate positions of all identified calbindin-negative (green, $n=40$ ) and calbindin-positive (blue, $n=16$ ) dopaminergic neurons, and whether their responses to a pinch stimulus (circles) or pinch and electrical stimuli (triangles) were tested or not (squares). Note that the calbindin-positive neurons were located mainly within the dorsal tier of $S \mathrm{NC}$. Calibration of ECOG and DA unit in $\boldsymbol{A}$ also applies to $\boldsymbol{B}$ and $\boldsymbol{C}$. ML (mediolateral) numbers in $\boldsymbol{C}$ denote positions with respect to midline. $\mathrm{R}$, Rostral; $C$, caudal; D, dorsal; $\mathrm{V}$, ventral.
SNc (Mann-Whitney $U$ tests, $p>0.05$ ). Thus, dopaminergic neurons in the $\mathrm{SNc}$ and $\mathrm{SNr}$ were grouped together (as dopaminergic substantia nigra neurons) for all further analyses.

The action potential waveforms of dopaminergic $\mathrm{SN}$ neurons filtered using standard bandpass settings of 300-5000 $\mathrm{Hz}$ (Fig. $1 A-C$ insets, Fig. $2 A$ ) exhibited a triphasic shape, with 24 of 56 neurons possessing a notch on the first ascending phase. Action potentials fired by identified dopaminergic SN neurons fitted the Ungless criteria established for identified dopaminergic VTA neurons (Ungless et al., 2004). Thus, the majority (53 of 56 neurons) exhibited biphasic action potential durations of $\geq 1.1 \mathrm{~ms}$ (Fig. $2 B, C$ ). Because standard bandpass filtering could in theory alter the shape of these extracellularly recorded action potentials, we also recorded unit activity using wideband filter settings of $0.3-5000 \mathrm{~Hz}$. Standard bandpass filter settings consistently resulted in a shorter biphasic action potential duration ( $1.36 \pm 0.02 \mathrm{~ms})$ when compared with the same action potentials filtered using wideband settings (biphasic duration $=1.73 \pm 0.05 \mathrm{~ms}, p<0.001$, Wilcoxon signed-rank test).

Previous work has shown that a population of nondopaminergic VTA neurons exhibit "dopamine neuron-like" characteristics; i.e., they have similar firing rates and firing patterns to dopaminergic VTA neurons (Ungless et al., 2004). However, in the current study, all labeled neurons that exhibited dopamine neuron-like electrophysiological characteristics expressed TH. Moreover, all labeled cells with a high firing rate $(>8 \mathrm{~Hz})$ and a biphasic action potential duration of $<1.1 \mathrm{~ms}$ were later verified neurochemically as nondopaminergic by the lack of TH immunoreactivity, and were always situated outside the SNc $(n=4$ neurons, data not shown).

\section{Activity of calbindin-negative and calbindin-positive dopaminergic neurons during cortical SWA}

Of 44 neurons recorded during cortical SWA, 30 were negative for calbindin immunoreactivity (CB-negative) (Fig. 1A) and 14 were positive (CB-positive) (Fig. $1 B$ ). On average, $\mathrm{CB}$-negative dopaminergic neurons fired action potentials at a significantly faster rate than CB-positive dopaminergic neurons (Fig. 3A). This difference was not associated with a difference in firing regularity (Fig. $3 A$ ), and was not attributable to a difference in the proportion of neurons exhibiting bursting activity according to the criteria described by Grace and Bunney (1984a) (percentage of CB-negative and CB-positive neurons firing bursts was $26.9 \%$ and $28.6 \%$, respectively). Furthermore, the proportions of CBnegative and $\mathrm{CB}$-positive dopaminergic neurons categorized as either pacemaker, random or bursty (Tepper et al., 1995) were similar (supplemental Fig. $1 D$, available at www.jneurosci.org as supplemental material). At the population level, neither CBnegative nor CB-positive dopaminergic neurons showed a preference for firing during either component of the slow cortical oscillation (Fig. 3B). The 3 neurons that did show a preference for firing in time with the cortical oscillation were CB-negative. Because different types of dopaminergic neurons can exhibit dis- 
tinct action potentials in vitro (Lammel et al., 2008; Margolis et al., 2008), we next tested whether this firing rate difference was mirrored by a difference in action potential waveforms in vivo. Regardless of filtering with either standard bandpass or wideband filter settings, the mean biphasic duration of the action potentials of CBnegative dopaminergic neurons was similar to that of CB-positive neurons (Fig. $2 B$ ). Under both filtering conditions, the distributions of action potential durations across the populations of CB-negative and CB-positive neurons were similar (Fig. $2 C)$. Standard bandpass filter settings produced a significantly shorter biphasic action potential duration than wideband filter settings for both CB-negative and CBpositive dopaminergic neuron groups ( $p<0.001$, Wilcoxon signed-rank test).

\section{Dopaminergic neuron activity during cortical SWA and the activated brain state}

Slow-wave activity was spontaneously interspersed with periods of cortical activation, characterized by a replacement of slow oscillations with smaller amplitude $(<200 \mu \mathrm{V})$, faster frequency $(>5 \mathrm{~Hz})$ heterogeneous activities. We recorded dopaminergic neuron activity during spontaneous fluctuations between these two extreme brain states, principally defined by either the presence or absence of the $\sim 1 \mathrm{~Hz}$ cortical slow oscillation. Twenty six dopaminergic neurons were recorded during only SWA, 12 dopaminergic neurons were recorded during only the cortical activation, and 18 neurons were recorded during both brain states.

Dopaminergic neurons recorded during SWA exhibited a mean firing rate similar to that of neurons recorded during the activated cortical state (Fig. 4). However, they altered their firing pattern in a brain state-dependent manner (Fig. $4 A, B$; supplemental Fig. $1 A, B$, available at www.jneurosci.org as supplemental material). Indeed, dopaminergic neurons recorded during SWA were, on average, significantly more irregular (as defined by CVs) than those neurons recorded during the activated cortical state (Fig. 4C). Moreover, the proportion of neurons that exhibited bursting activity (Grace and Bunney, 1984a) during SWA was higher than the proportion that fired bursts during the activated state (during SWA $27.5 \%$ of neurons fired bursts, compared with $12.5 \%$ of neurons in the activated state). When neurons were categorized as either pacemaker, random or bursty, most neurons recorded during SWA engaged in random firing. In agreement with our analysis of firing regularity as defined by $\mathrm{CVs}$, the distribution of neuron firing patterns became skewed in favor of pacemaker firing during the activated state (10 neurons classified as pacemaker, 2 as random and 2 as bursty; supplemental Fig. 1C, available at www.jneurosci.org as supplemental material). Of 30 dopaminergic neurons recorded during the activated brain state, 22 neurons were $\mathrm{CB}$-negative and 8 were $\mathrm{CB}$ positive. Importantly, during the activated brain state, $\mathrm{CB}$ negative neurons exhibited a significantly faster mean firing rate when compared with CB-positive dopaminergic neurons (mean firing rates $=3.57 \pm 0.28 \mathrm{~Hz}$ and $2.54 \pm 0.19 \mathrm{~Hz}$, respectively, Mann-Whitney $U$ test), but without a difference in mean firing regularity $(\mathrm{CB}$-negative $\mathrm{CV}=0.20 \pm 0.02, \mathrm{CB}$-positive $\mathrm{CV}=$ $0.17 \pm 0.03$, Mann-Whitney $U$ test) in agreement with unit activity seen during SWA.

\section{Responses of dopaminergic neurons to aversive stimuli}

We tested the responses of identified dopaminergic neurons to two standard types of aversive stimuli; a pinch and/or electrical stimulation of the hindpaw. Of the 44 identified dopaminergic neurons tested for their responses to a pinch stimulus, 30 were CB-negative, and 14 were CB-positive (Fig. 1D). Although the pinch stimulus consistently evoked an activated brain state, the responses of individual dopaminergic neurons were heterogeneous (Fig. $5 A, B$ ). Most CB-negative and CB-positive dopaminergic neurons did not respond to the stimulus (23 CB-negative and $11 \mathrm{CB}$-positive) (Fig. 5B). When neurons did respond, the most common response was an inhibition of firing (6 CBnegative and $2 \mathrm{CB}$-positive) (Fig. 5B). One CB-negative neuron exhibited an inhibition together with a rebound excitation and one CB-positive neuron exhibited a rebound excitation. The relative proportions of these heterogeneous responses within $\mathrm{CB}$ negative and CB-positive neuron groups did not differ (Fig. 5B).

The pooled responses of CB-negative and CB-positive dopaminergic neurons to the pinch stimulus showed no differences in the proportions of response types recorded when neurons were tested during SWA or the activated state (Fig. 5C). Similarly, there were no differences in the proportion of response types when CB-negative neurons were considered as a single group 
A Slow-Wave Activity

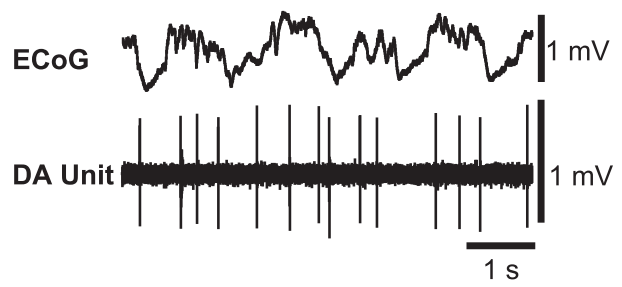

B

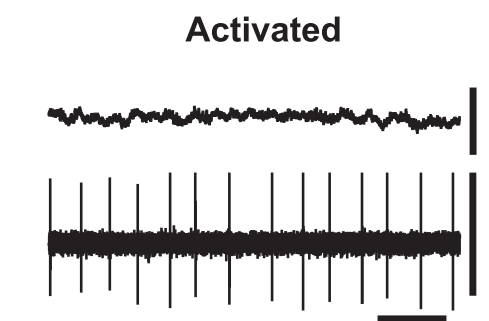

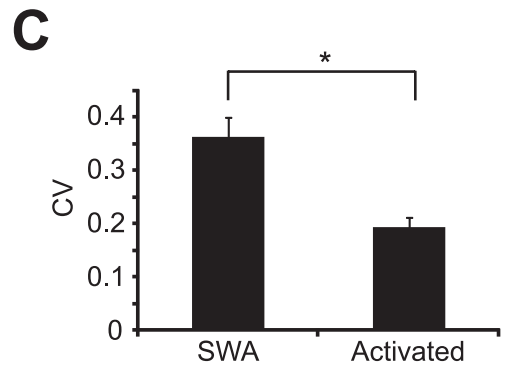

Figure 4. Identified dopaminergic neuron activity during two different brain states. $A$, Activity of an identified dopaminergic neuron (DA Unit) during SWA in the cortex (ECOG). Unit activity was irregular as shown by a flat AC. $B$, During the same recording session, and recording from the same neuron as in $A$, the firing pattern became markedly more regular during a spontaneous shift to an activated brain state [defined by the absence of the large $\sim 1 \mathrm{~Hz}$ cortical slow oscillation; see power spectra (pEC $0 \mathrm{G})$ ]. Note that peaks appeared in the $\mathrm{AC}$ at a similar time scale to the mean firing rate. $C$, Population analyses confirmed that neurons recorded during the activated brain state $(n=30)$ fired in a significantly more regular manner (lower $\mathrm{CV}$ ) than neurons recorded during SWA ( $n=44,{ }^{*} p<0.001$, Mann-Whitney $U$ test), whereas the firing rate did not change. Calibration in $A$ also applies to B. Data in C are means \pm SEMs.

(data not shown). The overall response of the dopaminergic neuron population to the pinch stimulus was a small but significant inhibition in firing rate during the stimulus (Fig. 5D). The weak nature of this inhibition at the population level was presumably because, at the single-cell level, most dopaminergic neurons were not responsive.

Of the 15 identified dopaminergic neurons tested for their responses to an electrical stimulus, 11 were CB-negative and 4 were CB-positive. In agreement with our pinch stimulus experiment, most dopaminergic neurons did not respond to the electrical stimulus (8 CB-negative and $4 \mathrm{CB}$-positive neurons were unresponsive) (Fig. 6A). The minority of CB-negative dopaminergic neurons that did respond $(n=3)$ exhibited a brief inhibition (response latency $=53.3 \pm 11.5 \mathrm{~ms}$ ) (Fig. $6 B$ ). The scarcity of significantly responding dopaminergic neurons was not likely the result of inefficacious stimulation because, in all cases, the electrical stimuli were associated with an evoked potential in the cortex (Fig. 6A,B). When pooled, the overall response of the dopaminergic neuron population to the electrical stimulus was a small but significant inhibition (Fig. 6C), similar to the response of the dopaminergic neuron population to a pinch stimulus.

\section{Discussion}

Here, we define the activity of identified subpopulations of dopaminergic SN neurons in vivo, during spontaneous and driven

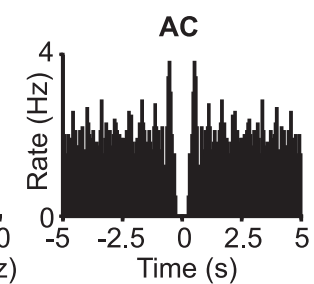

changes in brain state. We found that CBnegative dopaminergic neurons fire significantly faster than CB-positive dopaminergic neurons, and that this difference is not related to regularity of firing or bursting activity. Furthermore, activity of dopaminergic SN neurons is brain statedependent, and their firing pattern is significantly more irregular during SWA than during the activated brain state. Moreover, dopaminergic SN neurons respond heterogeneously to aversive somatosensory stimuli. Importantly, only a small proportion responded with an inhibition whereas most neurons did not respond.

\section{Activity of identified dopaminergic neurons in the substantia nigra during SWA}

Both CB-negative and CB-positive dopaminergic SN neurons typically exhibited slow spontaneous firing rates and regular and/or irregular firing patterns in vivo. This activity is in agreement with previous reports of presumed (Wilson et al., 1977; Grace and Bunney, 1983b; Celada et al., 1999; Hyland et al., 2002; Schultz, 2002) and intracellularly labeled (Grace and Bunney, 1980, 1983a) dopaminergic neurons recorded in vivo during unidentified brain states. Although the SNc contains nondopaminergic neurons (Nair-Roberts et al., 2008), we did not detect any nondopaminergic neurons that displayed dopamine neuron-like electrophysiological characteristics, as seen in the VTA (Ungless et al., 2004). Bursting activity was sparse, restricted to a small proportion of recorded neurons, and was of a lower level than reported for presumed dopaminergic VTA neurons (Shi, 2005; Zhang et al., 2008). These differences in bursting activity may be the result of different inputs to these two nuclei (Iribe et al., 1999; Paladini and Tepper, 1999; Blythe et al., 2007), differences in receptor expression (Keath et al., 2007), or differences in ongoing brain state (see below).

The majority of identified dopaminergic SN neurons did not fire in time with the cortical slow oscillation, which is notable when the activity of $\mathrm{SNc}$ afferents during the same brain state is considered. Because prominent excitatory afferents to the $\mathrm{SNc}$, namely the subthalamic nucleus (STN) (Smith et al., 1990) and the cholinergic neurons of the pedunculopontine nucleus (PPN) (Beninato and Spencer, 1988; Mena-Segovia et al., 2008b), fire predominantly in time with the active component of the cortical slow oscillation (Magill et al., 2001; Mena-Segovia et al., 2008b), these inputs appear unable to propagate this slow $\sim 1 \mathrm{~Hz}$ oscillation to dopaminergic SNc neurons. Of the principal inhibitory inputs to dopaminergic neurons, which include the striatum, $\mathrm{SNr}$ and globus pallidus (Hattori et al., 1975; Oertel et al., 1981; Smith and Bolam, 1989, 1990), only the striatum displays phasic activity correlated with cortical slow oscillations (Mallet et al., 2005). This suggests that neither rhythmic excitatory nor inhibitory inputs dominate dopaminergic SNc neuron firing patterns during SWA. 
Calbindin-negative and calbindinpositive dopaminergic neuron activity in vivo

Calbindin-negative dopaminergic neurons fired at higher rates than CB-positive dopaminergic neurons. It is uncertain whether calbindin-mediated calcium buffering is a critical determinant of these disparate activities, or simply a molecular correlate of diversity in other biophysical properties of dopaminergic neurons. Importantly, however, our findings in vivo agree with work in vitro demonstrating that CB-negative dopaminergic SN neurons tend to fire faster than CB-positive dopaminergic $\mathrm{SN}$ neurons (Neuhoff et al., 2002), a difference which primarily results from an augmented hyperpolarizationactivated cation current $\left(\mathrm{I}_{h}\right)$ in the former. Thus, diverse intrinsic membrane properties of these two subpopulations of dopaminergic neurons, particularly differential $I_{h}$ expression (Neuhoff et al., 2002), could underpin functional heterogeneity in vivo. This difference has important implications for Parkinson's disease, in which dopaminergic SN neurons degenerate and die. Indeed, CB-positive dopaminergic neurons are relatively well preserved in PD (Yamada et al., 1990). A lower firing rate, coupled with superior calcium buffering, would not only lessen metabolic demands on CB-positive neurons, but may enable them to cope better with the overexcitation and excitotoxic damage considered pivotal in PD pathology (Rodriguez et al., 1998). We observed no differences in the regularity of firing between CBnegative and $\mathrm{CB}$-positive dopaminergic neurons, or in the tendency of either of these groups to fire action potentials in bursts. Because the extents of both (single spike) firing irregularity and bursting activity in vivo are mostly attributable to inputs (Iribe et al., 1999; Paladini and Tepper, 1999; Blythe et al., 2007), the distribution and/or activity of the principal inputs to these dopaminergic subgroups are likely to be similar. Extracellularly recorded action potentials of CBnegative and $\mathrm{CB}$-positive dopaminergic neurons did not differ, suggesting that some intrinsic differences may only be uncovered using intracellular recordings (Neuhoff et al., 2002) and/or that calbindin has little bearing on action potential generation.

Dopaminergic neuron firing is brain state-dependent

Despite not firing on any particular component of the cortical slow oscillation, identified dopaminergic neuron firing patterns are brain state-dependent. When cortical SWA is absent, the $\sim 1$ $\mathrm{Hz}$ oscillatory firing in the PPN, STN and striatum is reduced (Magill et al., 2001; Mallet et al., 2005; Mena-Segovia et al.,
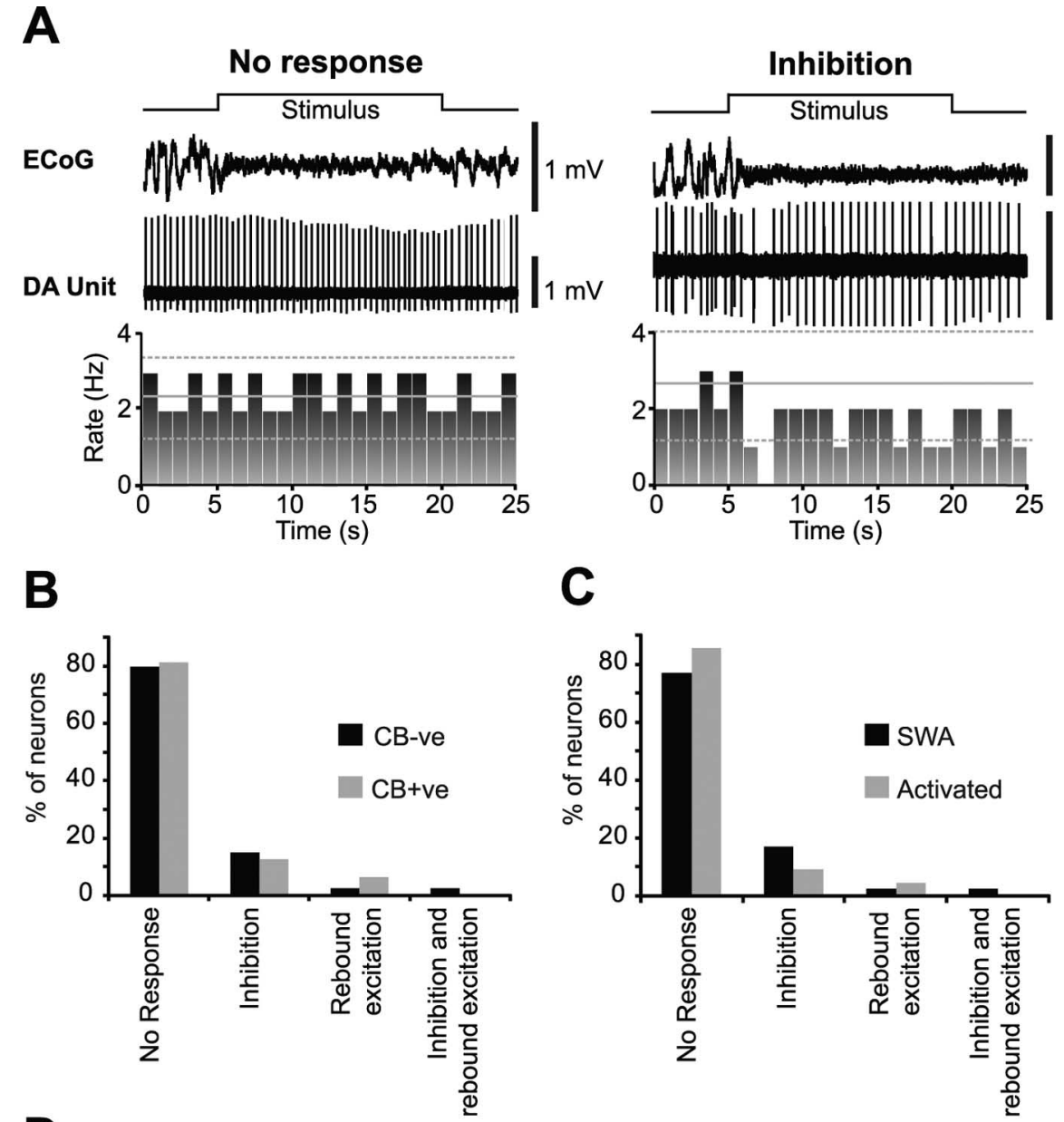

\section{C}
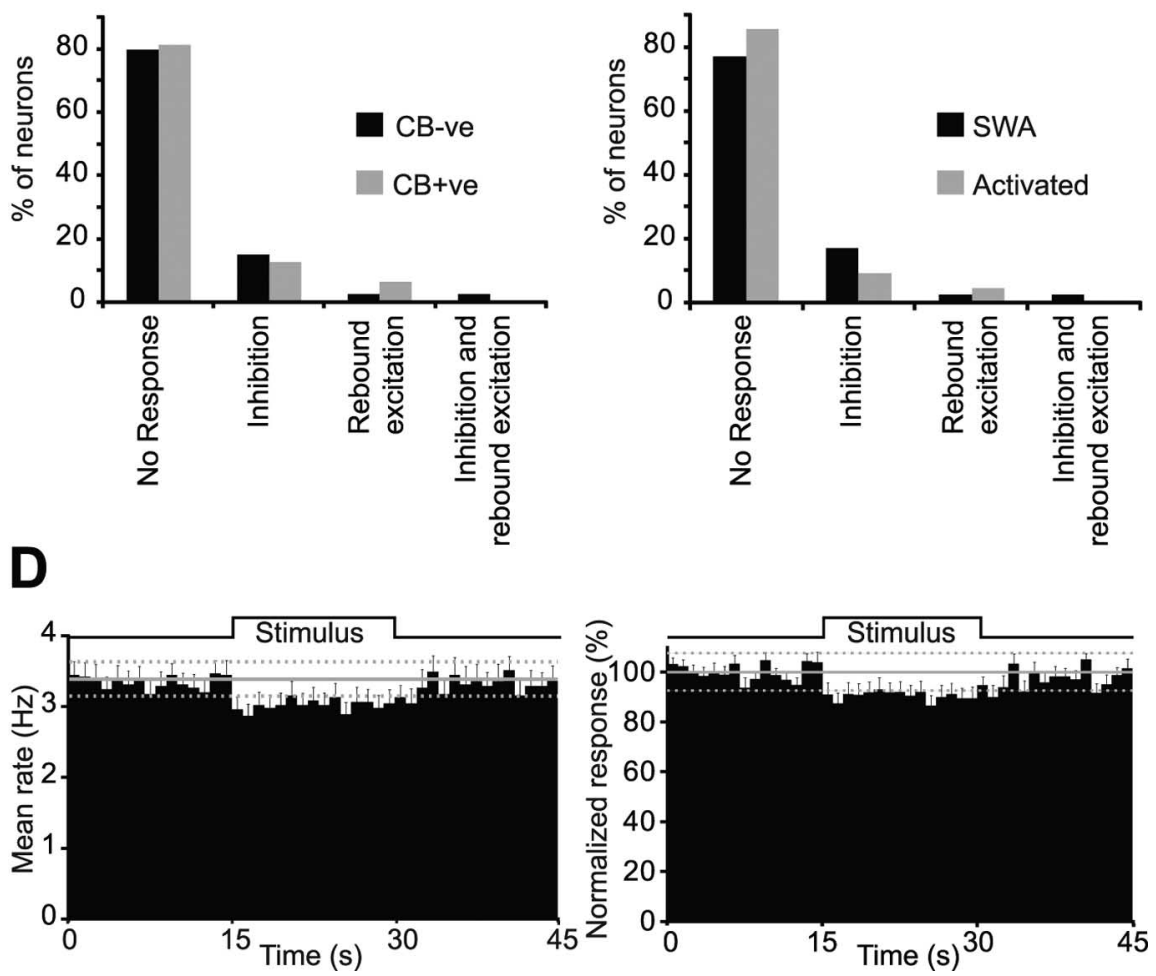

Figure 5. Individual and population responses of dopaminergic substantia nigra neurons to an aversive pinch stimulus. $\boldsymbol{A}$, Responses of individual dopaminergic neurons from $5 \mathrm{~s}$ before stimulus onset to $5 \mathrm{~s}$ after stimulus offset. Stimulus duration signaled by step up/down in line above ECoG. Firing rate histogram is plotted below unit activity. Note the evoked cortical activation present in both examples, confirming the efficacy of the stimulus. $\boldsymbol{B}, \boldsymbol{C}$, Main response types observed in the SN after a pinch stimulus were no response and inhibition. No differences were observed in the proportion of response types when neurons were grouped by either calbindin expression $(\boldsymbol{B})$ or by brain state $(\boldsymbol{C})\left(\chi^{2}\right.$ test). $\boldsymbol{D}$, When all neurons were pooled into a single population, a small but significant inhibition in response to the stimulus was observed, in terms of mean firing rate (left) and when data were normalized to baseline mean firing rate (right). Firing rate histograms in $\boldsymbol{A}$ and $\boldsymbol{D}$ are plotted in $1 \mathrm{~s}$ bins, with the baseline mean rate (solid gray line) \pm 2 SDs of this mean (dashed gray lines). Error bars in $\boldsymbol{D}$ indicate SEMs. 

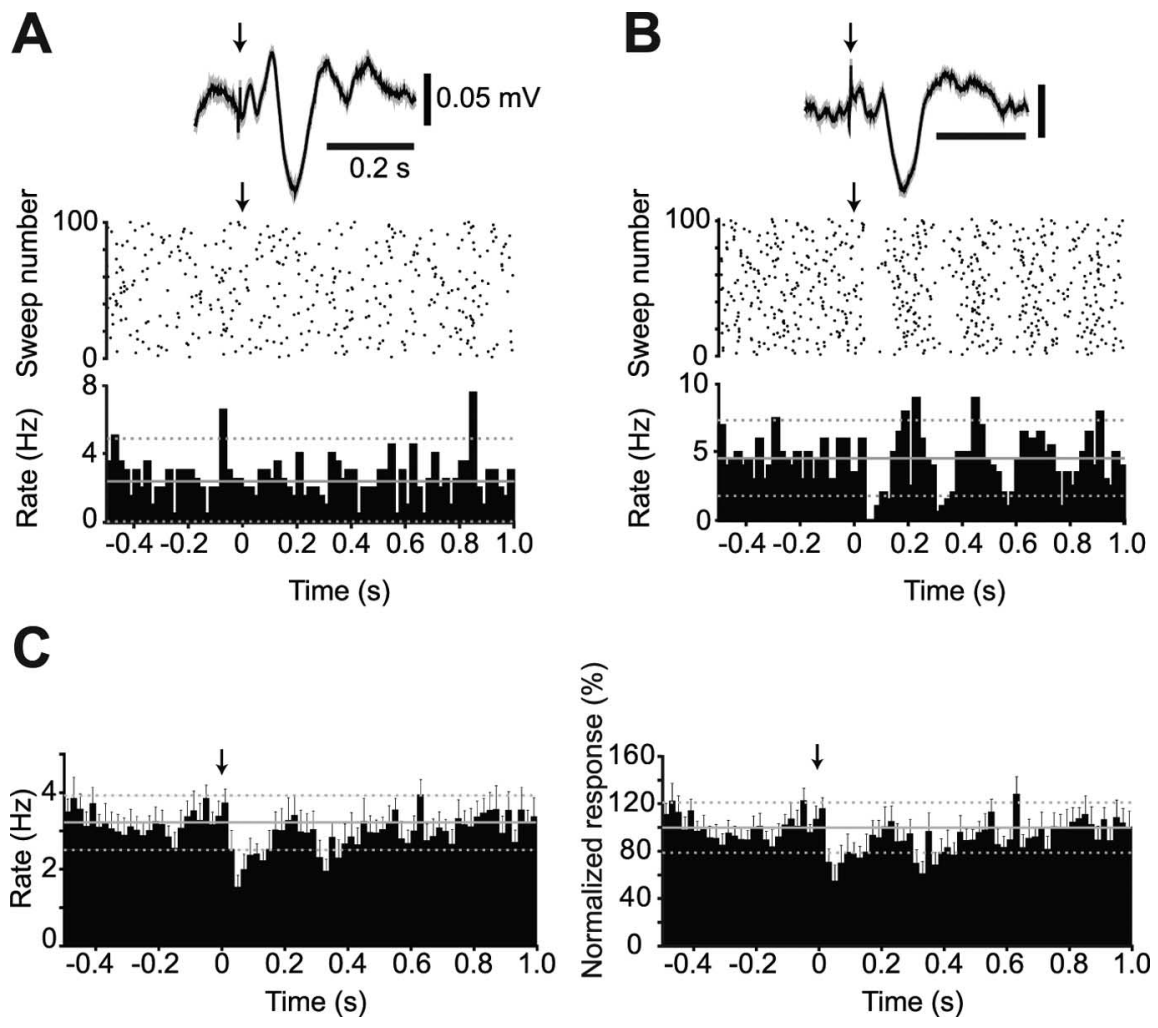

Figure 6. Individual and population responses of dopaminergic substantia nigra neurons to an aversive electrical stimulus. $\boldsymbol{A}$, PSTH and raster plot showing the activity of an individual dopaminergic neuron, which exhibited no response to the stimulus, from $0.5 \mathrm{~s}$ before to $1 \mathrm{~s}$ after stimulus delivery (denoted by arrow at time $=0$ ). Above is the evoked response in the cortex associated with the electrical stimulation, confirming stimulus efficacy. $\boldsymbol{B}$, PSTH and raster plot showing the inhibition of an individual dopaminergic neuron in response to stimulus delivery. $\boldsymbol{C}$, When all neurons were pooled into a single population, a small but significant inhibition in response to the electrical stimulus was observed, in terms of the mean firing rate (left), and when data were normalized to baseline mean rate (right). PSTHs in $\boldsymbol{A}-\boldsymbol{C}$ are plotted in $20 \mathrm{~ms}$ bins, with the baseline mean rate (solid gray line) \pm 2 SDs of this mean (dashed gray lines). Error bars in C indicate SEMs.

The mean firing rates of identified dopaminergic neurons recorded during SWA and cortical activation were similar. This agrees with observations of putative dopaminergic neurons recorded across the natural sleep-wake cycle (Steinfels et al., 1983), and with the finding that manipulating inputs to dopaminergic neurons can cause a change in firing pattern independent of firing rate (Tepper et al., 1995; Paladini et al., 1999; Paladini and Tepper, 1999). Furthermore, dopaminergic neurons tended to fire more bursts during SWA than during the activated state. This suggests that, during these different brain states, the spontaneously generated dopamine signal in the striatum is temporally distinct. Although the activated state during anesthesia mimics activity during natural waking, there is no perception or prediction during anesthesia, and we would expect more burst firing in behaving animals attending to salient stimuli (Hyland et al., 2002). Regardless, our data reiterate the importance of assessing ongoing brain state when recording from dopaminergic neurons.

\section{Dopaminergic $\mathrm{SN}$ neurons respond heterogeneously to aversive stimuli}

A key role of the midbrain dopaminergic system is one of rewarderror signaling within the basal ganglia; neurons within the VTA and $\mathrm{SNc}$ often show transient increases in activity during unpredicted rewards (Mirenowicz and Schultz, 1994; Schultz et al., 1997; Hyland et al., 2002). We have previously demonstrated that identified dopaminergic VTA neurons also show pronounced inhibitions of activity in response to an aversive somatosensory stimulus (Ungless et al., 2004). Our current data show that identified dopaminergic $\mathrm{SN}$ neurons are much less responsive to the same pinch stimulus than dopaminergic VTA neurons. Indeed, most dopaminergic SN neurons failed to respond to either the aversive pinch or electrical stimuli. Previous work has shown that similar pinch and electrical stimuli inhibit most unidentified, presumably dopaminergic SNc neurons (Tsai et al., 1980; Coizet et al., 2006). These discrepancies are not the result of ineffective pinch or electrical stimulation here because cortical activation and evoked potentials were consistently elicited, respectively, but might have arisen because of different anesthetic regimens. Ketamine, a NMDA receptor antagonist used here to supplement urethane anesthesia but not in the two previous studies, could have affected glutamatergic pathways influencing the transmission of somatosensory inputs that modulate dopaminergic neuron activity. However, because uniform inhibition of dopaminergic VTA neurons was elicited in the same anesthetic regimen as used here (Ungless et al., 2004), differences in the signaling of aversive events in VTA and SN are directly comparable. Moreover, given VTA receives a larger glutamatergic innervation than SNc (Smith et al., 1996), ketamine should have proportionally greater impact on VTA responses, making this difference particularly remarkable. Importantly, our finding that inhibition is not the default response of dopaminergic SN neurons to aversive stimuli mirrors previous work in monkeys (Schultz and Romo, 1987; Joshua et al., 2008).

In conclusion, we demonstrate another key functional difference between dopaminergic SN neurons and dopaminergic VTA neurons, and suggest that proportionally fewer dopaminergic SN neurons are recruited during signaling of specific aversive events. Differing responses to external stimuli may be the result of different inputs to these two dopaminergic neuron populations, or different receptor expression that underlies varying sensitivity to the same afferent projections. Because no significant differences were observed in the proportions of CB-negative and CB-positive dopaminergic SN neurons responding to the stimuli, it is likely that extrinsic inputs to these two SN neuron populations do not differ. Inhibition of midbrain dopaminergic neuron activity in response to aversive stimuli is of importance for the reward-error prediction hypothesis. Although our data do not challenge this hypothesis, they suggest that, unlike dopaminergic VTA neurons, the default response of dopaminergic SN neurons to aversive stimuli is not inhibition when a prediction cannot be made, or when the aversive qualities cannot be perceived.

\section{References}

Balcita-Pedicino JJ, Sesack SR (2007) Orexin axons in the rat ventral tegmental area synapse infrequently onto dopamine and gammaaminobutyric acid neurons. J Comp Neurol 503:668-684.

Beninato M, Spencer RF (1988) The cholinergic innervation of the rat sub- 
stantia nigra: a light and electron microscopic immunohistochemical study. Exp Brain Res 72:178-184.

Björklund A, Dunnett SB (2007) Dopamine neuron systems in the brain: an update. Trends Neurosci 30:194-202.

Blythe SN, Atherton JF, Bevan MD (2007) Synaptic activation of dendritic AMPA and NMDA receptors generates transient high-frequency firing in substantia nigra dopamine neurons in vitro. J Neurophysiol 97:2837-2850.

Brown P, Kupsch A, Magill PJ, Sharott A, Harnack D, Meissner W (2002) Oscillatory local field potentials recorded from the subthalamic nucleus of the alert rat. Exp Neurol 177:581-585.

Cahusac PM, Morris R, Salt TE, Hill RG (1990) Sensory responses of caudal trigeminal neurons to thermal and mechanical stimuli and their behavioural correlates in the rat. Neuroscience 36:543-551.

Cameron DL, Wessendorf MW, Williams JT (1997) A subset of ventral tegmental area neurons is inhibited by dopamine, 5-hydroxytryptamine and opioids. Neuroscience 77:155-166.

Celada P, Paladini CA, Tepper JM (1999) GABAergic control of rat substantia nigra dopaminergic neurons: role of globus pallidus and substantia nigra pars reticulata. Neuroscience 89:813-825.

Coizet V, Dommett EJ, Redgrave P, Overton PG (2006) Nociceptive responses of midbrain dopaminergic neurones are modulated by the superior colliculus in the rat. Neuroscience 139:1479-1493.

Fallon JH, Moore RY (1978) Catecholamine innervation of the basal forebrain. IV. Topography of the dopamine projection to the basal forebrain and neostriatum. J Comp Neurol 180:545-580.

Fallon JH, Riley JN, Moore RY (1978) Substantia nigra dopamine neuronsseparate populations project to neostriatum and allocortex. Neurosci Lett 7:157-162.

Gerfen CR, Baimbridge KG, Miller JJ (1985) The neostriatal mosaic: compartmental distribution of calcium-binding protein and parvalbumin in the basal ganglia of the rat and monkey. Proc Natl Acad Sci U S A $82: 8780-8784$.

Gerfen CR, Baimbridge KG, Thibault J (1987) The neostriatal mosaic: III. Biochemical and developmental dissociation of patch-matrix mesostriatal systems. J Neurosci 7:3935-3944.

Gonzalez-Hernandez T, Rodriguez M (2000) Compartmental organization and chemical profile of dopaminergic and GABAergic neurons in the substantia nigra of the rat. J Comp Neurol 421:107-135.

Grace AA, Bunney BS (1980) Nigral dopamine neurons: intracellular recording and identification with L-dopa injection and histofluorescence. Science 210:654-656.

Grace AA, Bunney BS (1983a) Intracellular and extracellular electrophysiology of nigral dopaminergic neurons-2. Action potential generating mechanisms and morphological correlates. Neuroscience 10:317-331.

Grace AA, Bunney BS (1983b) Intracellular and extracellular electrophysiology of nigral dopaminergic neurons-1. Identification and characterization. Neuroscience 10:301-315.

Grace AA, Bunney BS (1984a) The control of firing pattern in nigral dopamine neurons: burst firing. J Neurosci 4:2877-2890.

Grace AA, Bunney BS (1984b) The control of firing pattern in nigral dopamine neurons: single spike firing. J Neurosci 4:2866-2876.

Grenhoff J, Ugedo L, Svensson TH (1988) Firing patterns of midbrain dopamine neurons: differences between A9 and A10 cells. Acta Physiol Scand 134:127-132.

Hattori T, Fibiger HC, McGeer PL (1975) Demonstration of a pallido-nigral projection innervating dopaminergic neurons. J Comp Neurol 162:487-504.

Hyland BI, Reynolds JN, Hay J, Perk CG, Miller R (2002) Firing modes of midbrain dopamine cells in the freely moving rat. Neuroscience 114:475-492.

Iribe Y, Moore K, Pang KC, Tepper JM (1999) Subthalamic stimulationinduced synaptic responses in substantia nigra pars compacta dopaminergic neurons in vitro. J Neurophysiol 82:925-933.

Joshua M, Adler A, Mitelman R, Vaadia E, Bergman H (2008) Midbrain dopaminergic neurons and striatal cholinergic interneurons encode the difference between reward and aversive events at different epochs of probabilistic classical conditioning trials. J Neurosci 28:11673-11684.

Keath JR, Iacoviello MP, Barrett LE, Mansvelder HD, McGehee DS (2007) Differential modulation by nicotine of substantia nigra versus ventral tegmental area dopamine neurons. J Neurophysiol 98:3388-3396.

Korotkova TM, Sergeeva OA, Eriksson KS, Haas HL, Brown RE (2003) Ex- citation of ventral tegmental area dopaminergic and nondopaminergic neurons by orexins/hypocretins. J Neurosci 23:7-11.

Lammel S, Hetzel A, Hackel O, Jones I, Liss B, Roeper J (2008) Unique properties of mesoprefrontal neurons within a dual mesocorticolimbic dopamine system. Neuron 57:760-773.

Magill PJ, Bolam JP, Bevan MD (2000) Relationship of activity in the subthalamic nucleus-globus pallidus network to cortical electroencephalogram. J Neurosci 20:820-833.

Magill PJ, Bolam JP, Bevan MD (2001) Dopamine regulates the impact of the cerebral cortex on the subthalamic nucleus-globus pallidus network. Neuroscience 106:313-330.

Mallet N, Le Moine C, Charpier S, Gonon F (2005) Feedforward inhibition of projection neurons by fast-spiking GABA interneurons in the rat striatum in vivo. J Neurosci 25:3857-3869.

Mallet N, Pogosyan A, Marton L, Bolam JP, Brown P, Magill PJ (2008) Parkinsonian beta oscillations in the external globus pallidus and their relationship with subthalamic nucleus activity. J Neurosci 28:14245-14258.

Margolis EB, Mitchell JM, Ishikawa J, Hjelmstad GO, Fields HL (2008) Midbrain dopamine neurons: projection target determines action potential duration and dopamine $\mathrm{D}_{2}$ receptor inhibition. J Neurosci 28:8908-8913.

Mena-Segovia J, Winn P, Bolam JP (2008a) Cholinergic modulation of midbrain dopaminergic systems. Brain Res Rev 58:265-271.

Mena-Segovia J, Sims HM, Magill PJ, Bolam JP (2008b) Cholinergic brainstem neurons modulate cortical gamma activity during slow oscillations. J Physiol 586:2947-2960.

Mirenowicz J, Schultz W (1994) Importance of unpredictability for reward responses in primate dopamine neurons. J Neurophysiol 72:1024-1027.

Mirenowicz J, Schultz W (1996) Preferential activation of midbrain dopamine neurons by appetitive rather than aversive stimuli. Nature 379:449-451.

Nair-Roberts RG, Chatelain-Badie SD, Benson E, White-Cooper H, Bolam JP, Ungless MA (2008) Stereological estimates of dopaminergic, GABAergic and glutamatergic neurons in the ventral tegmental area, substantia nigra and retrorubral field in the rat. Neuroscience 152:1024-1031.

Neuhoff H, Neu A, Liss B, Roeper J (2002) $I_{\mathrm{h}}$ channels contribute to the different functional properties of identified dopaminergic subpopulations in the midbrain. J Neurosci 22:1290-1302.

Oertel WH, Schmechel DE, Brownstein MJ, Tappaz ML, Ransom DH, Kopin IJ (1981) Decrease of glutamate decarboxylase (GAD)-immunoreactive nerve terminals in the substantia nigra after kainic acid lesion of the striatum. J Histochem Cytochem 29:977-980.

Paladini CA, Tepper JM (1999) GABA(A) and GABA(B) antagonists differentially affect the firing pattern of substantia nigra dopaminergic neurons in vivo. Synapse 32:165-176.

Paladini CA, Celada P, Tepper JM (1999) Striatal, pallidal, and pars reticulata evoked inhibition of nigrostriatal dopaminergic neurons is mediated by GABA(A) receptors in vivo. Neuroscience 89:799-812.

Paxinos G, Watson C (1986) The rat brain in stereotaxic coordinates. Sydney: Academic.

Pinault D (1996) A novel single-cell staining procedure performed in vivo under electrophysiological control: morpho-functional features of juxtacellularly labeled thalamic cells and other central neurons with biocytin or Neurobiotin. J Neurosci Methods 65:113-136.

Pollard M (2000) Ionotropic glutamate receptor-mediated responses in the rat primary somatosensory cortex evoked by noxious and innocuous cutaneous stimulation in vivo. Exp Brain Res 131:282-292.

Prensa L, Parent A (2001) The nigrostriatal pathway in the rat: A singleaxon study of the relationship between dorsal and ventral tier nigral neurons and the striosome/matrix striatal compartments. J Neurosci 21:7247-7260.

Redgrave P, Prescott TJ, Gurney K (1999) Is the short-latency dopamine response too short to signal reward error? Trends Neurosci 22:146-151.

Rodriguez MC, Obeso JA, Olanow CW (1998) Subthalamic nucleusmediated excitotoxicity in Parkinson's disease: a target for neuroprotection. Ann Neurol 44:S175-S188.

Schultz W (2002) Getting formal with dopamine and reward. Neuron $36: 241-263$.

Schultz W, Romo R (1987) Responses of nigrostriatal dopamine neurons to high-intensity somatosensory stimulation in the anesthetized monkey. J Neurophysiol 57:201-217. 
Schultz W, Romo R (1990) Dopamine neurons of the monkey midbrain: contingencies of responses to stimuli eliciting immediate behavioral reactions. J Neurophysiol 63:607-624.

Schultz W, Dayan P, Montague PR (1997) A neural substrate of prediction and reward. Science 275:1593-1599.

Shi WX (2005) Slow oscillatory firing: a major firing pattern of dopamine neurons in the ventral tegmental area. J Neurophysiol 94:3516-3522.

Smith Y, Bolam JP (1989) Neurons of the substantia nigra reticulata receive a dense GABA-containing input from the globus pallidus in the rat. Brain Res 493:160-167.

Smith Y, Bolam JP (1990) The output neurones and the dopaminergic neurones of the substantia nigra receive a GABA-containing input from the globus pallidus in the rat. J Comp Neurol 296:47-64.

Smith Y, Hazrati LN, Parent A (1990) Efferent projections of the subthalamic nucleus in the squirrel monkey as studied by the PHA-L anterograde tracing method. J Comp Neurol 294:306-323.

Smith Y, Charara A, Parent A (1996) Synaptic innervation of midbrain dopaminergic neurons by glutamate-enriched terminals in the squirrel monkey. J Comp Neurol 364:231-253.

Steinfels GF, Heym J, Strecker RE, Jacobs BL (1983) Response of dopaminergic neurons in cat to auditory stimuli presented across the sleepwaking cycle. Brain Res 277:150-154.
Steriade M (2000) Corticothalamic resonance, states of vigilance and mentation. Neuroscience 101:243-276.

Steriade M, Nunez A, Amzica F (1993) A novel slow ( $<1 \mathrm{~Hz})$ oscillation of neocortical neurons in vivo: depolarizing and hyperpolarizing components. J Neurosci 13:3252-3265.

Tepper JM, Martin LP, Anderson DR (1995) GABA receptor-mediated inhibition of rat substantia nigra dopaminergic neurons by pars reticulata projection neurons. J Neurosci 15:3092-3103.

Tsai CT, Nakamura S, Iwama K (1980) Inhibition of neuronal activity of the substantia nigra by noxious stimuli and its modification by the caudate nucleus. Brain Res 195:299-311.

Ungless MA, Magill PJ, Bolam JP (2004) Uniform inhibition of dopamine neurons in the ventral tegmental area by aversive stimuli. Science 303:2040-2042.

Wilson CJ, Young SJ, Groves PM (1977) Statistical properties of neuronal spike trains in the substantia nigra: cell types and their interactions. Brain Res 136:243-260.

Yamada T, McGeer PL, Baimbridge KG, McGeer EG (1990) Relative sparing in Parkinson's disease of substantia nigra dopamine neurons containing calbindin-D28K. Brain Res 526:303-307.

Zhang D, Yang S, Jin GZ, Bunney BS, Shi WX (2008) Oscillatory firing of dopamine neurons: differences between cells in the substantia nigra and ventral tegmental area. Synapse 62:169-175. 